\title{
Application of a Susceptible, Infectious, and/or Recovered (SIR) Model to the COVID-19 Pandemic in Ecuador
}

\author{
Pablo Espinosa ${ }^{1}$, Paulina Quirola-Amores ${ }^{2}$ and Enrique Teran ${ }^{1,2 *}$ \\ ${ }^{1}$ Colegio de Ciencias de la Salud, Universidad San Francisco de Quito, Quito, Ecuador, ${ }^{2}$ Instituto de Microbiología, Colegio de \\ Ciencias Biológicas y Ambientales-COCIBA, Universidad San Francisco de Quito, Quito, Ecuador
}

The coronavirus disease 2019 (COVID-19) pandemic is wreaking havoc in healthcare systems worldwide. COVID-19 was reported for the first time in Wuhan (China) and the first case in Ecuador was confirmed on February 27, 2020. Several determinants are taken into consideration for the establishment of asymptomatic or critical illness, and are necessary to predict the dynamics and behavior of a pandemic. We generated a Susceptible, Infectious, and/or Recovered model and reflected upon the COVID-19 pandemic in Ecuador. For the entire Ecuadorian population, we estimated that the reproduction number $(R 0)$ was 2.2, with $88 \%$ susceptible/infected individuals. To stop a national epidemic, a quarantine for 3-4 months is required, and when 55\% of the population has been immunized (equivalent to 110 days since the first report of a COVID-19 case), a real decrease of new cases will be

OPEN ACCESS

Edited by:

Hui-Jia Li,

Beijing University of Posts and Telecommunications (BUPT), China

Reviewed by: Wen-Xuan Wang,

Beijing University of Posts and Telecommunications (BUPT), China Liangli Yang,

University of Science and Technology Beijing, China

*Correspondence: Enrique Teran eteran@usfq.edu.ec

Received: 11 June 2020 Accepted: 24 September 2020 Published: 30 November 2020

Citation:

Espinosa P, Quirola-Amores $P$ and Teran E (2020) Application of a Susceptible, Infectious, and/or Recovered (SIR) Model to the COVID-

19 Pandemic in Ecuador.

Front. Appl. Math. Stat. 6:571544.

doi: 10.3389/fams.2020.571544 observed. The effectiveness of quarantine should be analyzed retrospectively, and not as a result of contemporary control of the COVID-19 epidemic.

Keywords: R0, COVID-19, herd immunity, susceptible infected-recovered model, attack rate

\section{INTRODUCTION}

The coronavirus disease 2019 (COVID-19) pandemic that is wreaking havoc in healthcare systems worldwide is caused by severe acute respiratory syndrome coronavirus 2 (SARS-CoV-2) infection. Several phylogenetic studies have determined the origin of this zoonotic virus and, simultaneously, the potential reservoirs and amplifying species (especially mammals) [1-3].

The first report of SARS-CoV-2 infection was in December 2019 in Wuhan (Hubei Province, China). Worldwide spread of SARS-CoV-2 was inevitable due to its easy dissemination (as droplets), apparently low mortality, and high incidence of asymptomatic cases ( $\geq 15.8 \%$ of infected people) [1-5].

In $<3$ months, $60 \%$ of countries reported COVID-19 cases, particularly in Europe (e.g., Italy, Spain). Then, SARS-CoV-2 infection was documented in North America and, one week later, in Brazil, Ecuador, and Chile.

For COVID-19, lethality has been estimated to be $4-6 \%$, mortality to be $1-1.5 \%$, and severe illness to occur in $\geq 4.7 \%$ of diagnosed cases. The recovery time has been estimated to range from 8 to 11.5 days, but it is related to multiple factors [6-8].

The Ministry of Public Health (MSP) of Ecuador reported the first case of COVID-19 on February 27, 2020. It was an Ecuadorian woman who returned from Spain to Guayaquil on a commercial flight and had a welcoming party. Then, other cases at the northern (Tulcan) and southern (Machala) borders appeared (arising potentially from Colombia and Peru, respectively) [8]. By the beginning of 
June, according to official reports, there were $\geq 43,120$ confirmed cases, with a higher prevalence in people aged 21-60 years, lethality of $4-7 \%$, and a mortality rate $(<1 \%)[8,9]$.

Mathematical models such as the Susceptible, Infectious, and/ or Recovered (SIR) model are used to predict different scenarios related to epidemiologic factors and possible outcomes to assess epidemic spread. The reproduction number $(R 0)$ is used to estimate the capacity of viral transmission from one person to another. The effective reproduction number ( $R e$ or $R t$ ) refers to the number of infected people in a specific time interval based on the $R 0$. The susceptible fraction (Fso) takes into account the percentage of infected people that might exist in an epidemic. These indices show how a population and a virus are related to make predictions and consider the consequences at a given moment $[8,10,11]$.

We aimed to generate SIR models based on data provided by the Ecuadorian MSP to estimate R0, Rt, Fso, time in quarantine, and the required percentage of immunized people to consider a reduction in the total incidence of COVID-19 in Ecuador. This strategy would allow the creation of a new SIR model readily without the need to have a large volume of regional data or national data. The possibility of developing retrospective and predictive models in this or other conditions based on an $R 0$ calculation (observed here as a dynamic value) and the generation of a dynamic $R t$ value that will serve as a reference to make decisions in real-time, along with another type of model for quarantine and immunization, would be very helpful.

\section{METHODOLOGY}

\subsection{Data Source}

The data used for these models were from daily reports produced by the MSP. The data comprised of cumulative cases, new cases, excluded cases, deceased cases, cases who recovered from COVID-19, number of testing samples, and samples already collected but waiting to be processed from day-1 to day 30 of the epidemic (https://www.gestionderiesgos.gob.ec/informes-desituacion-covid-19-desde-el-13-de-marzo-del-2020/) [9]. Values were taken from day 1 to day 30 of the epidemic considering the range with the highest reliability of data.

The SIR model, predictions/estimations, and tabulation data were processed using Excel $_{\mathrm{TM}}$ within Office ${ }_{\mathrm{TM}}$ v16.37 (Microsoft, Redmond, WA, United States). Model parameters were verified using Vensim тм v8.0.9 (Salisbury, United Kingdom) and RStudioтм v4.0 (R Foundation for Statistical Computing, Vienna, Austria). Graphs were generated using Prism тм $_{\text {v8.4.2 }}$ (GraphPad, San Diego, CA, United States).

\subsection{Estimated Infection Rate for the COVID-19 Epidemic in Ecuador}

We used the parameters related to the number of individuals infected per day, the trend of the epidemic, mean number of samples processed, and samples waiting to be processed to determine the percentage of "true" work carried out by the MSP. Then, we compared the number of daily infected cases and estimated the rate of increase (cases/day). This was done using Eq. 1, in which the numerator is the number of reported cases in a final period of time and the denominator is the number of reported cases in an initial time $[12,13]$.

$$
\begin{aligned}
\text { rate of increase }\left[\frac{\text { cases }}{\text { day }}\right] & =\frac{\text { Infected } t 2}{\text { Infected } t 1} \\
\text { contagion rate } & =(1-\text { rate of increase }) * 100 \%
\end{aligned}
$$

The result using Eq. 1 revealed whether the number of cases increased or not from 1 day to another, and also predicted the number of new cases for the next day. Thus, it also estimated the daily rate of contagion (Eq. 2), and the percentage of contagion in a certain period, based on the rate of increase. Both rates enabled the observation of dynamic behavior with $R 0$ and $R t$.

\subsection{Calculation of the Minimum Number of Cases Required to Generate the COVID-19 Epidemic in Ecuador}

A mathematical model based on sigmoid curves was applied to estimate the minimum number of cases required to generate an epidemic ("community infection"). To calculate the constants for the equation, two methods were employed. First, Eq. 3 (K1) was obtained from the curve for the number of positive case reported by the MSP using logarithmic regression, where $t$ is the time of infection, and $I$ is the number of people infected in time $t$. Second, Eq. $4(K 2)$ was considered as a differential logarithm regarding time, where $T i$ is the initial infection time, $T f$ is the final infection, If denotes finally-infected individuals and $I i$ denotes initiallyinfected people in an estimated period of time [14].

$$
\begin{aligned}
K 1 & =\frac{\sum \operatorname{Ln}(t)-\operatorname{Ln}(I)-\frac{\sum \operatorname{Ln}(t) * \sum L b(I)}{n}}{\sum(\operatorname{Ln}(t))^{2}-\frac{\sum(\operatorname{Ln}(t))^{2}}{n}} \\
K 2 & =\frac{\operatorname{Ln}(I f)-\operatorname{Ln}(I i)}{T f-T i}
\end{aligned}
$$

For $\mathrm{K} 1$ all the study data was used and in $K 2$ data were from shorter ranges. Then, $K 2$ provides daily evolution while $K 1$ provides a general view of the epidemic. To calculate It (Eq. 5) (the number of infections over time in an epidemic), Ic was the number of infections present when the epidemic started. Based on the results, K1 (Eq. 3) was 0.1972, and K2 (Eq. 4) was 0.2386.

$$
I t=\left[\frac{1}{1+I c e^{-k t}}\right] I c^{2}
$$

\subsection{Duration of Quarantine}

To determine the duration of quarantine and its subsequent removal, we needed to calculate the recovery time of the population. Hence, we took the minimum number of cases after the first recovery of COVID-19, and a hypothetical daily peak of infections (for our purpose this was considered to be 100 daily cases [15]) and a threshold of cases after this peak for considering lifting the quarantine. Hence, when this number of infected cases was reached, with no increase in the last few days, 
quarantine could be lifted. We assumed that all the daily reports from the MSP detailed the maximum number of cases, so we would know how long it would take for the recovery of these infected individuals [14].

$$
\begin{aligned}
I t & =\frac{I f}{1+(I f-R e o) e^{-k I f t}} \\
k & =\operatorname{Ln}\left[\frac{\frac{I f}{I t}+1}{I f}\right] \\
t d & =\frac{\operatorname{Ln}\left[\frac{I f}{\frac{I f}{I f}}\right]}{k}
\end{aligned}
$$

In Eq. 6, It is the number of recoveries in a period, If is the maximum number of infections in a particular period, Reo is the number of people who recovered on day- 0 after reaching the maximum number of infected individuals (usually 1). In Eq. 7, $k$ is the constant of proportionality. In Eq. 8, $t d$ is the duration of quarantine, $I r$ is the maximum number of infected people minus the threshold of 100 infected individuals [15] needed for lifting the quarantine $(I r=I f-100)$ and $I f$ is the maximum of cases for each day of quarantine.

\subsection{SIR Models for COVID-19}

We wished to model and predict the behavior of the COVID-19 epidemic in Ecuador. Hence, we took into account three mathematical models based on SIR, differed in the use of different parameters for the equations and the calculation of $R 0$, where we did not consider the rate of asymptomatic cases (not a well-established value at the time of analyses).

The first model (Eqs 9-16) was based on the number of infections generating new cases. The parameters for construction were the recovery rate $(95 \%)$, mortality rate $(5 \%)$, time needed for recovery (17 days), $R 0$ (taken from international databases [5], and assuming that $40 \%$ of the population was under total quarantine (that is equivalent to $4 \%$ less probability of becoming infected). $N$ was the total population of Ecuador and the $R 0$ was the ratio between the number of infected people and the number of people who recovered and those who died (Eqs 13, 16) [13, 16-18].

$$
\begin{aligned}
\% P & =\frac{N C o M}{I i T D S i} \\
C o M & =\frac{I i T D S i}{N \% P} \\
R e & =I i *\left(\frac{T R}{D I}\right) \\
M & =I i *\left(\frac{T M}{D I}\right) \\
R 0 & =\frac{C o M}{(R e+M)} \\
R t & =\frac{C o M(t)}{(\operatorname{Re}(t)+M(t))} \\
d R t & =R 0 *\left(\frac{1}{1+e^{\left(\frac{S o}{N}\right)}}\right) *\left(\frac{S o}{N}\right) *\left(e^{\left(\frac{S o}{N}\right)}\right) \\
R 0 m & =\sqrt[n]{R 01 * R 02 * R 0 n}
\end{aligned}
$$

Sf (Eq. 17) represents the number of vulnerable individuals, If (Eq. 23) is the number of infected people, $R f$ (Eq. 21) is the number of people who recovered from COVID-19, $M f($ Eq. 22) is the number of people who died; all of these parameters were taken after a certain period of time. DI represents the duration of illness in days, $T D$ (Eq. 10) is the percentage of infections $(R 0), \% P$ (Eq. 9) is the probability of becoming infected, TR (Eq. 11) is the percentage of people who recovered, TM (Eq. 12) is the percentage of individuals who died, and Rt (Eqs 12, 15) is the $R 0$ that varies through time and the function of infected people.

The second model (Eqs 17-26) takes into consideration differential equations from a standard model that were integrated subsequently with national data where the percentage of people who recovered $(\gamma)$ (Eq. 17) was $95 \%$, mortality ( $\mu$ ) (Eq. 22) was $5 \%$, and the time for recovery (TR) (Eqs 9, 11, 20) was 17 days. To calculate $R 0$ for Ecuador, we considered standard calculations (Eqs 21, 26). $R t$ (Eqs 14, 15, 24) was based on the variation in the percentage of susceptible people during a particular time, and $p$ (Eq. 17) was the relationship between the number of people who recovered from COVID-19 and the infection rate $[13,19]$.

$$
\begin{aligned}
p & =\frac{\gamma}{\beta} \\
p & =\frac{N-S f}{\operatorname{Ln}\left(\frac{\gamma N-\gamma R}{\gamma S o}\right)} \\
R 0 & =\frac{\beta}{\gamma} S o \\
S f & =S o e^{-\left[\frac{N-S o}{p}\right]} \\
R f & =\left(\frac{\gamma}{T R}\right) R o \\
M f & =\mu I i \\
I f & =S f=I i-R i-M i \\
R t & =R o * e^{\left(\frac{S o}{N}\right)} \\
d R t & =R 0 *\left(\frac{1}{1+e^{\left(\frac{S o}{N}\right)}}\right) *\left(\frac{S o}{N}\right) * e^{\left(\frac{S o}{N}\right)} \\
R 0 m & =\sqrt[n]{R 01 * R 02 * R 0 n}
\end{aligned}
$$

The third model (Eqs 27-36) was based on the infection rate calculated with the equation for the rate of increase, so the $\beta$ value (Eq. 27) was assigned to it. For the recovery rate $(\gamma)$, it was considered that 17 days was the minimum time required to recover, and a $3 \%$ mortality percentage $(\mu)$ in accordance to the MSP report $[10,13]$.

$$
\begin{aligned}
\beta & =1-\text { rate } \\
\gamma & =\frac{1}{\text { recuperation time }} \\
R 0 & =\frac{\beta}{(\gamma+\mu)} \\
\text { Infection (Con) } & =\beta * I i * R 0 *\left(\frac{\text { So }-I i}{N}\right)
\end{aligned}
$$




$$
\begin{aligned}
& \text { If }=\text { So }- \text { Con }-R e-M u \\
\operatorname{Recuperation}(R e)= & I f * \gamma \\
\operatorname{Dead}(M u)= & I f * \mu \\
R t & =R o * e\left(\frac{S o}{N}\right) \\
d R t & =R 0 *\left(\frac{1}{1+e^{\left(\frac{S o}{N}\right)}}\right) *\left(\frac{S o}{N}\right) *\left(e^{\left(\frac{S o}{N}\right)}\right) \\
R 0 m & =\sqrt[n]{R 01 * R 02 * R 0 n}
\end{aligned}
$$

\subsection{Variation of the SIR Model With Different Values of $R 0$}

Taking into consideration the first proposed SIR model (Eqs 9-16), we generated different $R 0$ values (Eqs 13, 16) and observed their behavior during a particular time. This strategy allowed for the ascertainment of how $R t$ and the epidemic cycle were related so that we could observe it and identify possible "bottlenecks", when the number of susceptible people decreased (for whatever reason). Simultaneously, we compared the derivative of Rt (Eq. 15), which allowed us to observe the maximum number of infections when $R t$ varied, and worked to adjust a national $R 0$ [14]. For model validation, we applied a difference between the curve for infected people (from the MSP report) and the number of infections generated with the three SIR models. Then, the curve (of these three models) that best fits to the real data reported by the MSP, was selected for further analysis [14].

\subsection{Susceptible Factors and the Minimum Percentage Required for Immunization Using Rt}

For the Rt of each model, we calculated the susceptible factor (Fso) (Eq. 37). This strategy allowed us to observe the highest percentage of infections (based on the variation in $R t$ ) to generate a threshold that estimated the direction of the epidemic and where $R 0$ was located in Ecuador [10, 19]. With Fso and $R 0$ generated in each model, we could predict the minimum percentage (\%Pi,v) (Eq. 38) required for immunization (by vaccination or herd immunity) to stop COVID-19 spread [10, 19].

$$
\begin{aligned}
& f S o(t)=1-e^{-R t} \\
& \% P i, v=1-\frac{1}{R 0}
\end{aligned}
$$

\subsection{Vaccination and Immunization}

After the derivation of $\% P i, v$ from Eq. 38, we considered the incorporation of the $R t$ toward the population $(N)$. Considering the variation in infection, we could recognize how long it would take for a vaccination strategy to obtain results and stop infection. We could also observe the number of immunized individuals and those who would not be infected during a particular time [10, 18, 20].

$$
\text { Immunizate population }(\mathrm{Piz})=N *\left(1+\left(\frac{1}{2 R t^{2}}\right)\right)
$$

$$
\text { Infected population }(P i f)=N-N *\left(1+\left(\frac{1}{2 R t^{2}}\right)\right)
$$

\section{RESULTS}

Upon analyses of all the reported data by the MSP, a marked delay in sample processing was noticed $(40 \% \mathrm{CI}= \pm 0.0035)$ and an increase in the number of new daily cases of $28.5 \%(C I= \pm 0.00146)$ was reported. Hence, the infection curve was delayed in $28.5 \%$ of cases ( $40 \%$ of undetermined cases). This observation was confirmed when the rate of increase per day remained the average between the number of delayed samples and number of reported cases. This resulted in 1.20 reported cases per day $(\mathrm{CI}=$ \pm 0.0103 ). When we analyzed the number of cases with COVID-19 reported by the MSP, there was a 30\% delay in confirmatory diagnose. This problem was most evident at day 33 of the epidemic when there was $60 \%$ delay in confirmatory diagnoses (Figure 1).

We used $K$ values $($ Eqs 3, 4) to determine the minimum number of cases required to generate an epidemic in Ecuador. In our simulation using $K 1$, five cases were needed for an epidemic in Ecuador. Also, the model using K2 showed that with six cases, an exponential communitarian infection was possible (Figure 2), and that recovery from COVID-19 could take 18 days $(\mathrm{CI}= \pm 0.0945)$.

While observing the trend in the increase in infected cases, one key question was how long the quarantine should last until there were 100 infected cases. We calculated that quarantine should be lifted after approximately $80-110$ days $(\mathrm{CI}= \pm 0.2001)$ because the maximum number of cases had been reached (Figure 3).

Simultaneously with obtaining results, we generated three SIR models with variation of some parameters to obtain $R 0$ and observed which model was the best fit.

When the number of infected people was compared for each model, model 1 (SIR 1) obtained the closest values to the true situation. Hence, we chose model 1 to observe the dynamic in cases once quarantine had been implemented (Figure 4). Using this model, $R 0=2.2(\mathrm{CI}=-1.644,+2.75)$. Importantly, with each model, an $R 0$ value was generated so a different $R t$ analysis was undertaken to detect the appearance of a bottleneck in a susceptible population. We found that, for each model, the $R 0$ was between 1 and $3(\mathrm{CI}=-1.865,+1.974)$ (Table 1).

Using these values, we calculated the maximum number of people susceptible to SARS-CoV-2 infection for each $R 0$ value $(95.02 \%, C I= \pm 1.27)$. The same calculation showed that a maximum of $66.67 \%(\mathrm{CI}= \pm 2.70)$ of the same population would need to be immunized to stop the epidemic (Figure 5).

When quarantine parameters were generated with SIR 1 , the peak of the maximum number of infected individuals would be reached on day 154 of the epidemic (65 more days than if quarantine was not applied). The number of new cases would decrease $(64.6 \%, \mathrm{CI}= \pm 2.35)$ but not disappear, demonstrating that when the quarantine time increased, the number of infected cases was redistributed. This phenomenon was observed when the different $R t$ values (generated by a variation in parameters) 


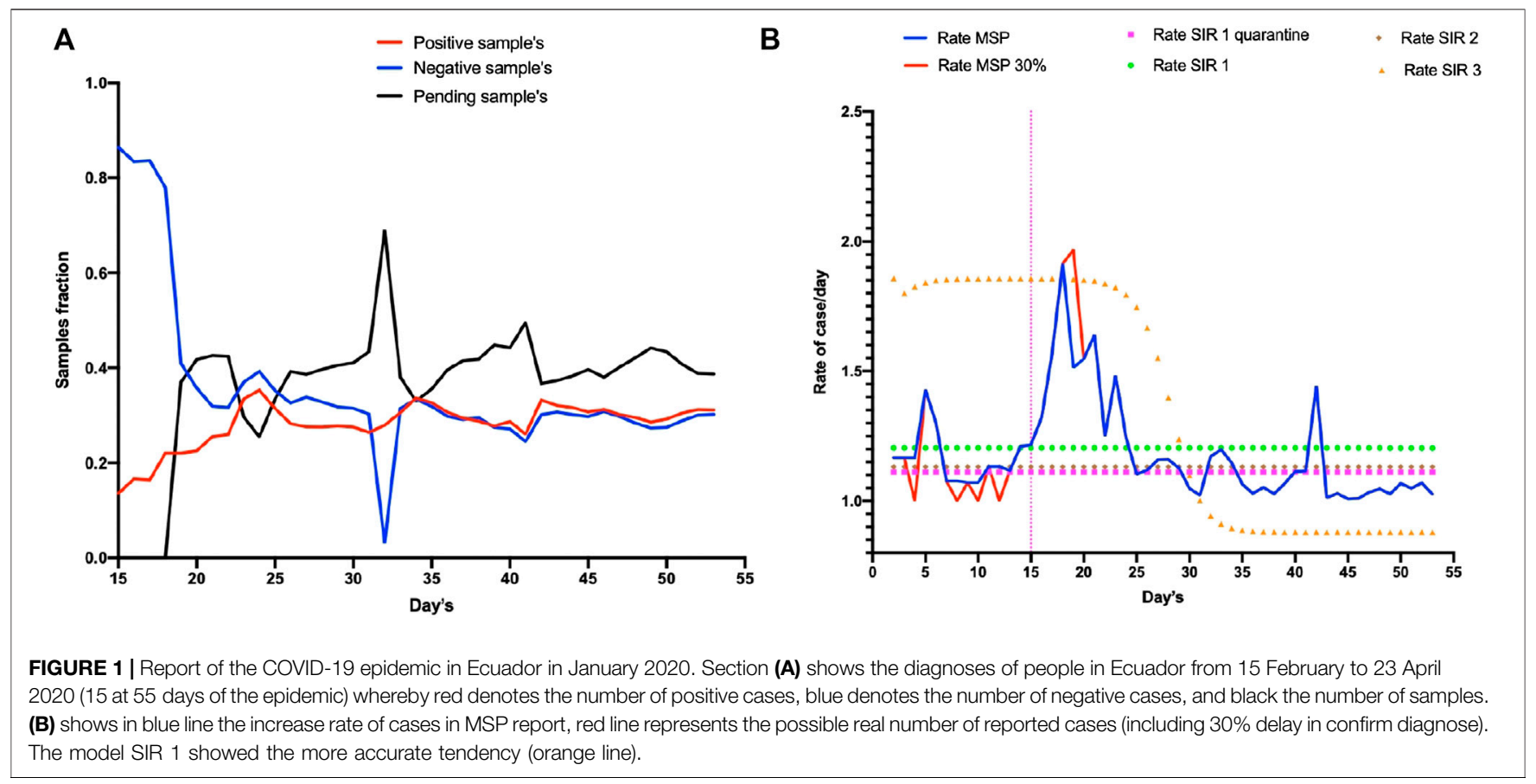

were analyzed (Figure 6) and demonstrated that, for each unit increase in $R t$, the epidemic would increase by 10 days $(\mathrm{CI}=$ \pm 0.295 ).

To confirm the obtained $R 0$ with SIR 1 , we analyzed the variation in $R t$ with its derivative, in addition to the variation in
Fso with regard to $R t$. As the bottleneck appeared with $R t$, without considering quarantine, the value of $R t$ remained in this bottleneck demonstrating that, in reality, $R 0$ was between 1.5 and $2.5 \mathrm{CI}=-1.51,+2.48$. When comparing these data with Fso, we found that the maximum number of cases using this $R 0$ value

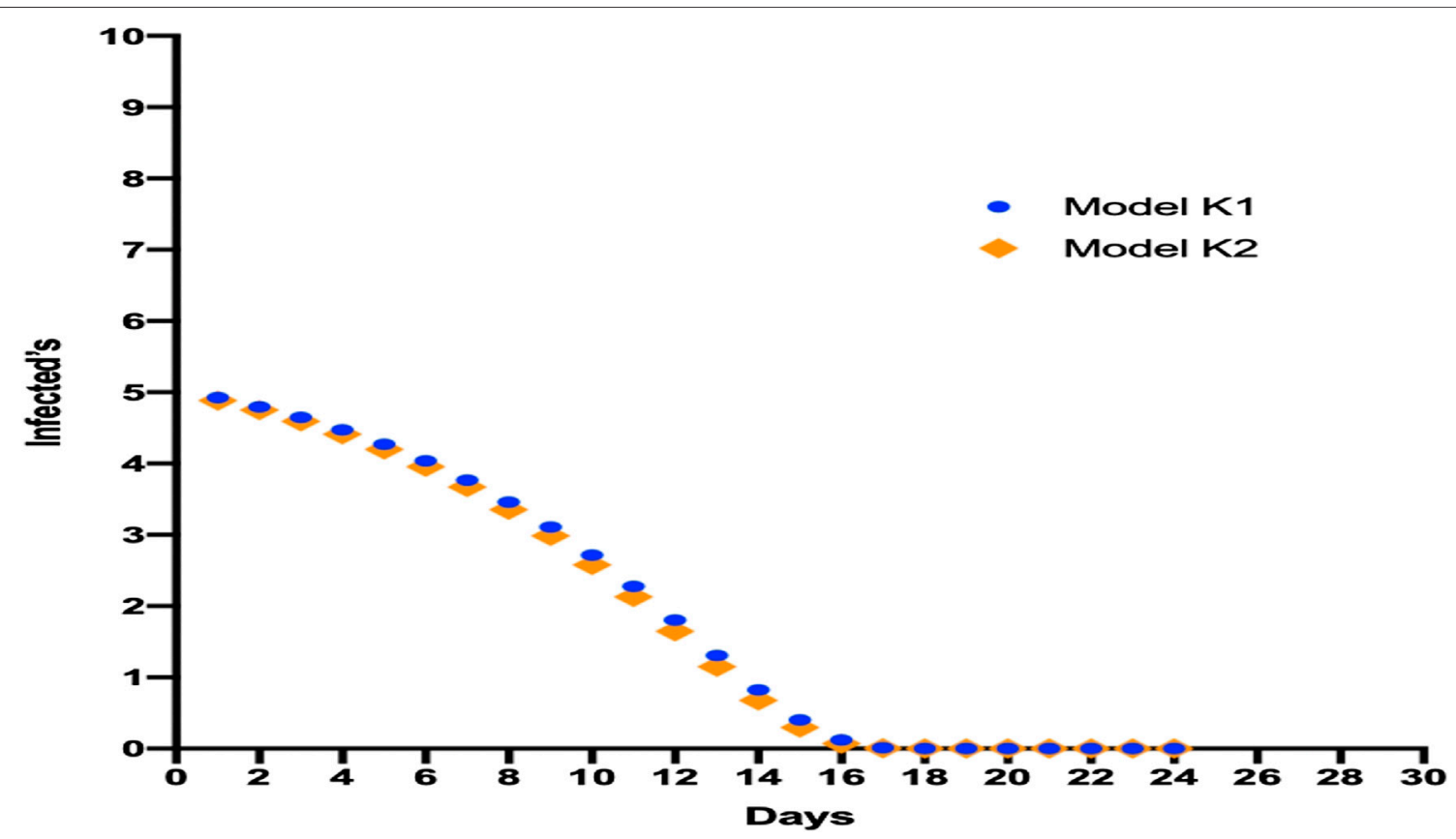

FIGURE 2 | Model for the minimum number of cases required for an epidemic in Ecuador showing different simulations with various $\mathrm{K}$ constants for observing this number, communitarian infection, and the average time needed for recovery. 


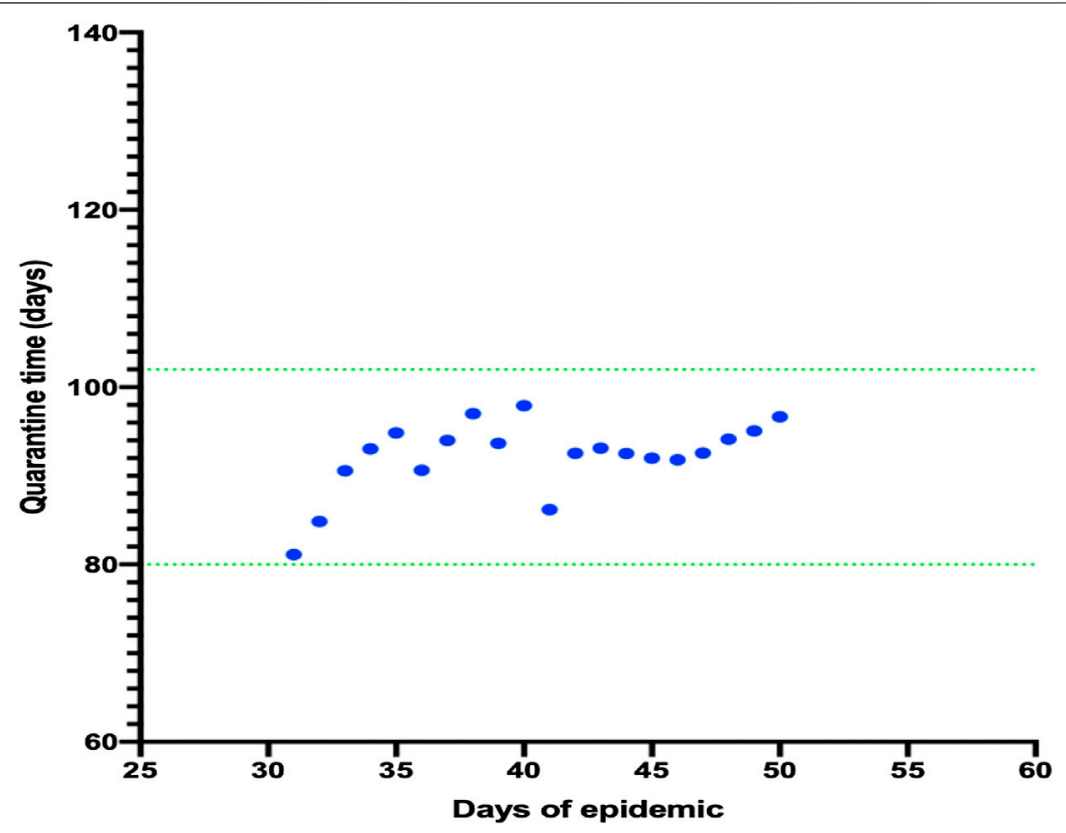

FIGURE 3 | Model for the number of days required for quarantine and the maximum number of daily cases. It was observed that the number of days necessary to finish the quarantine remained the same despite the increasing number of cases daily.

was $80 \%$, with a maximum time of 90 days (when the epidemic would begin to decrease). However, if quarantine was applied, this value reached $86 \%$ of infected people at a time of 140 days (Figure 7).
Upon application of all the different data of $R 0$, an issue was observed when an immunization appeared (even for vaccination or herd immunity). The most important question is how long will it take to observe if this strategy is successful. Our model

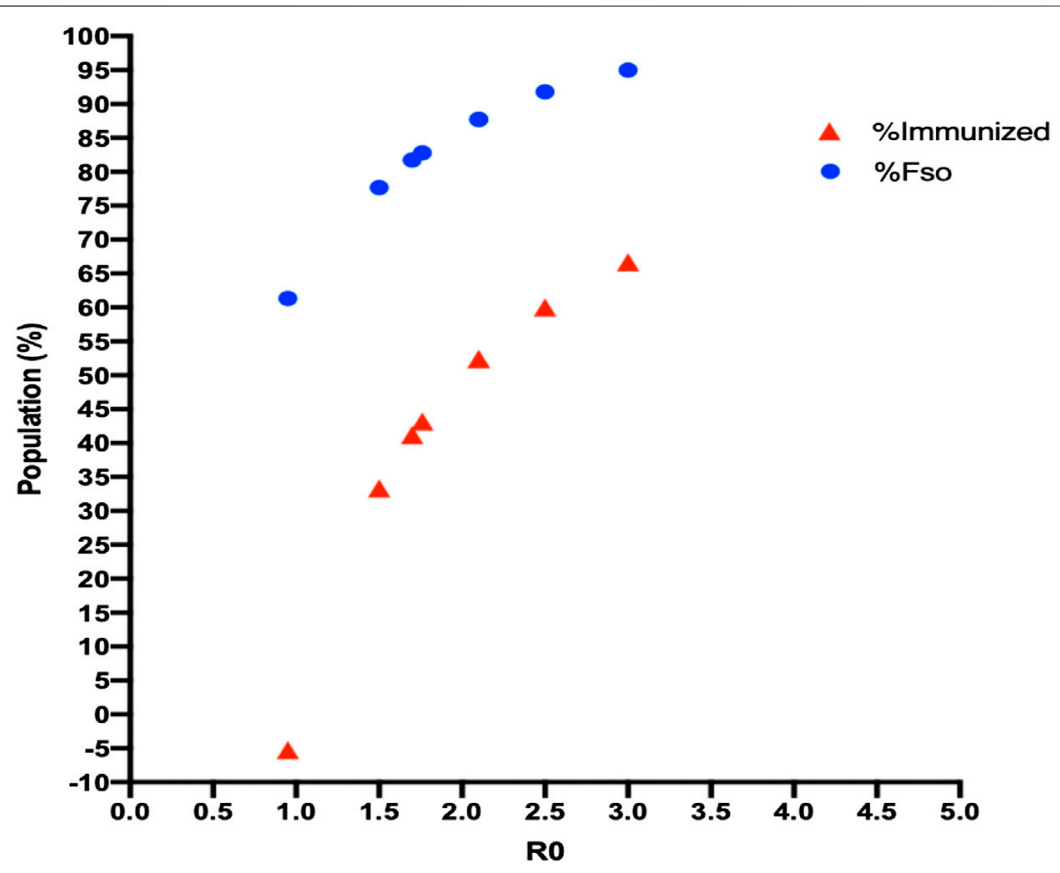

FIGURE 4 | Fso and herd-immunity values with $R 0$ for each model. An increase in $R 0$ for each SIR model led to an increase in the infected population and herd immunization, but neither of these values reached $100 \%$ of the population. 
TABLE 1 | $R 0$ values generated with three SIR models showing the maximum number of people who may be infected (Fso), and herd immunity for each model.

\begin{tabular}{lcccc}
\hline & $\boldsymbol{R} \mathbf{0}$ & $\boldsymbol{R 0 m}$ & Fso (\%) & Herd immunity (\%) \\
\hline R0 Ref. 28 & 2.1 & 2.1 & 87.75 & 52.3 \\
SIR 1 & 1.73 & $2.20(\mathrm{Cl}=-1.644,+2.75)$ & 81.73 & 41.18 \\
& 3 & & 95.02 & 66.67 \\
SIR 2 & 2.1 & & 87.75 & 52.38 \\
& 1.76 & 1.92 & 82.80 & 43.18 \\
SIR 3 & 2.1 & $(\mathrm{Cl}=-1.865,+1.974)$ & 87.75 & 52.38 \\
& 0.95 & 1.19 & 61.33 & -5.26 \\
& 1.5 & $(\mathrm{Cl}=-1.042,+1.337)$ & 77.69 & 33.33
\end{tabular}

$\mathrm{ROm}$ is the average of the RO value calculated in each model.

showed that, without quarantine from day-80 to day 100 , the introduction of vaccination, the rebound of immunized individuals, and a decrease in the number of infected cases applying quarantine between day 100 to day 120 would result in the same outcome (Figure 8).

\section{DISCUSSION}

The model used in our study is not the only model to predict the behavior of COVID-19. One must consider the dynamic of SARSCoV-2 transmission, availability of resources, and delay in diagnosis when choosing the best model. For Ecuador, an important issue was the large number of people with pending SARS-CoV-2 tests. This factor, together with asymptomatic individuals and underreporting of "true" infected people (by consensus, it is 55\%), demonstrated why our model was not well-fitted with the data reported by the MSP $[9,23]$.
Although the estimated time needed for a patient to recover from COVID-19 was 18-20 days, some scholars have stated it is $8-10$ days using SIR models. According to the MSP, it takes 20 days, which poses problems for healthcare units in terms of space and resources [5, 23, 24]. Determining this value is a key component in the prediction based on SIR models because it is based on local data. Subsequently, extrapolations can be made in the hope that they are not too far from reality.

In our study, the time for recovery from COVID-19 was based on a $K$ model and observing how the recovery curve was adjusted to the true situation. Mistakes can be generated because of the issues mentioned above as well as from the reports of patients who have recovered from COVID-19. Even though this value cannot be observed directly, it can be estimated through the report of deaths by the MSP and comparing them with the registry of the National Institute of Statistics of Ecuador. This comparison resulted in a difference of $\geq 20 \%$ and has been observed in other studies too [23-26].

Through an assessment of the first 100 cases of COVID-19, a marked difference in acceleration and control of the epidemic was made in Taiwan. The Taiwan government isolated and quarantined all individuals related to each COVID-19 case, and tracked all asymptomatic individuals. This strategy showed that, according to the proposed model, quarantining the first six cases rapidly and aggressively would have created a different scenario from that seen presently but, as the author mentions, the switch from theory to practice is not easy [25]. Another important observation is the rate of increase of cases. At the national level, the rate of increase was constant (the number of cases increased in 5-7 days), as has been observed on a global scale $[9,27]$. Hence, locally obtained data were a reflection of

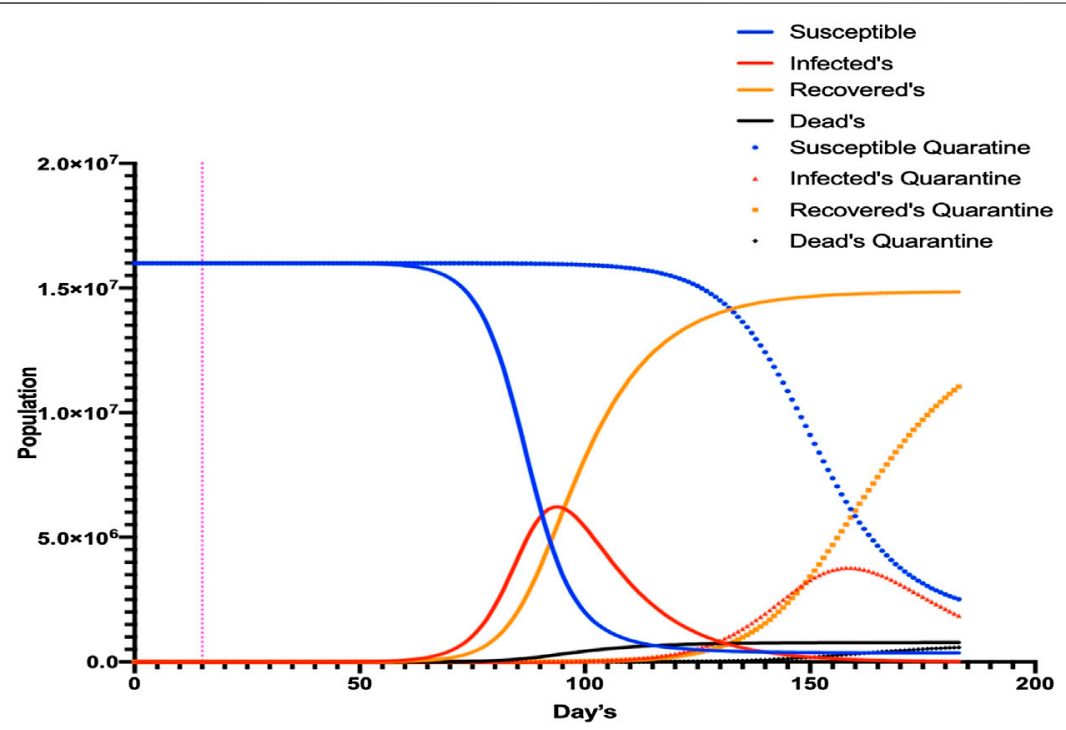

FIGURE 5 | SIR1 model with modified parameters for quarantine. Different simulations were undertaken for COVID-19, with and without quarantine. The number of infected individuals decreased but the duration of infectivity was prolonged by $>200$ days. 


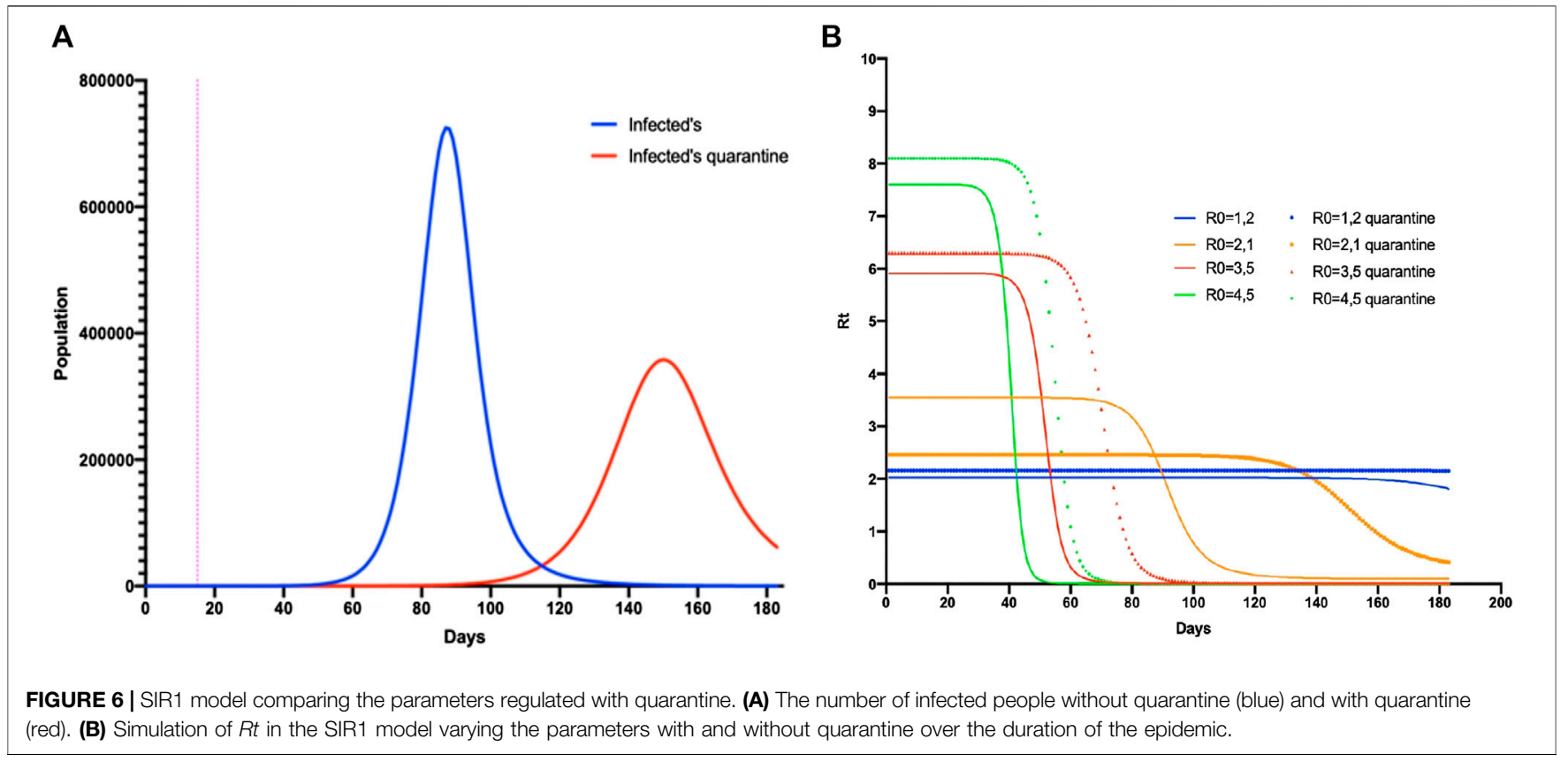

what occurred in the past when SARS-CoV-2 spread in Asia and Europe $[9,27]$.

Our model estimated that lifting the quarantine should occur approximately 2-3 months after the peak of infection has been reached. In China, after the peak of infection (after 17 days), the quarantine was lifted during day 13 to day 14 . Such lifting is dependent upon time and the capacity of the health system of a particular country. In addition, a strict quarantine works not only by holding back the appearance of new cases, but also allows the health system to recover and prepare for new cases $[5,27]$.

The mortality rate for COVID-19 has been estimated to be about $3-7 \%$, and results in another problem for SIR models. In our model, we took a mortality rate of $5 \%$ (in other models, it varies between 3 and 4.5\%). However, one must consider other causes of death unrelated to COVID-19 that can affect a model indirectly $[5,23,24,29]$. The increase in $R 0$ determines how effective the strategies taken by local authorities are. In our study,

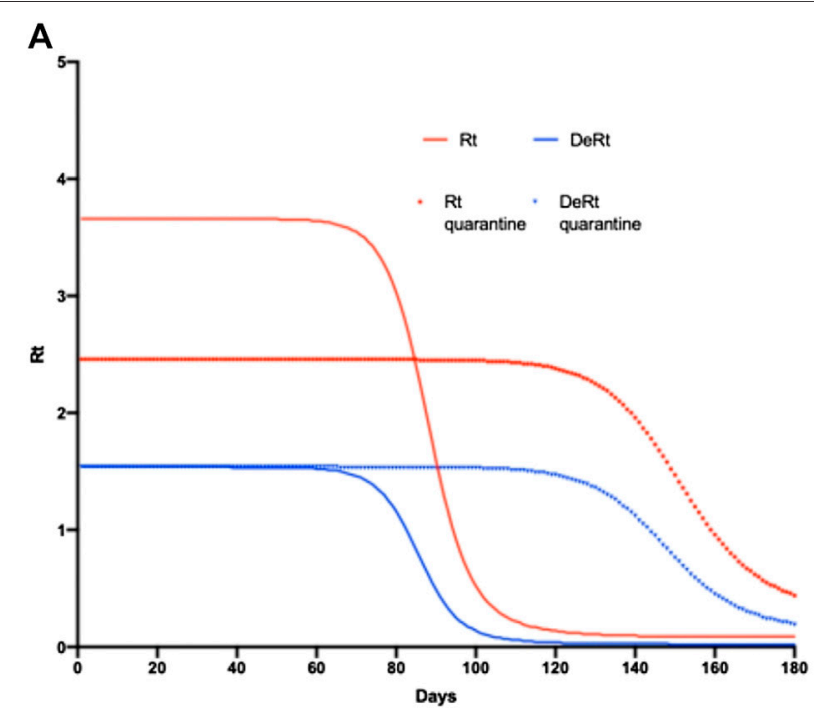

B

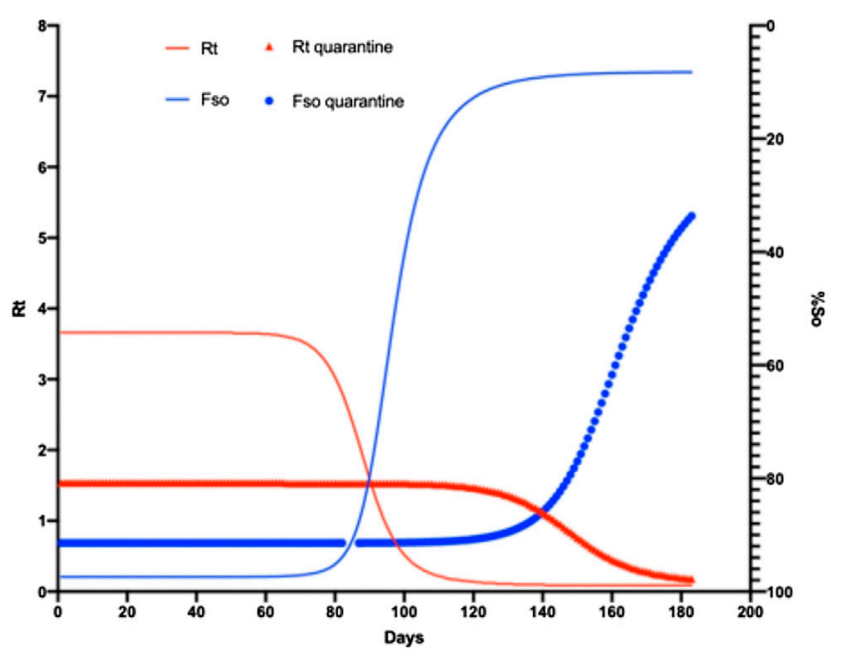

FIGURE 7 | SIR1 model comparing regular parameters with quarantine. In (A) the simulation of Rt is observed in the SIR1 model varying the parameters with and without quarantine throughout the epidemic duration. (B) shows the number of infections without quarantine (blue) and with quarantine (red). 


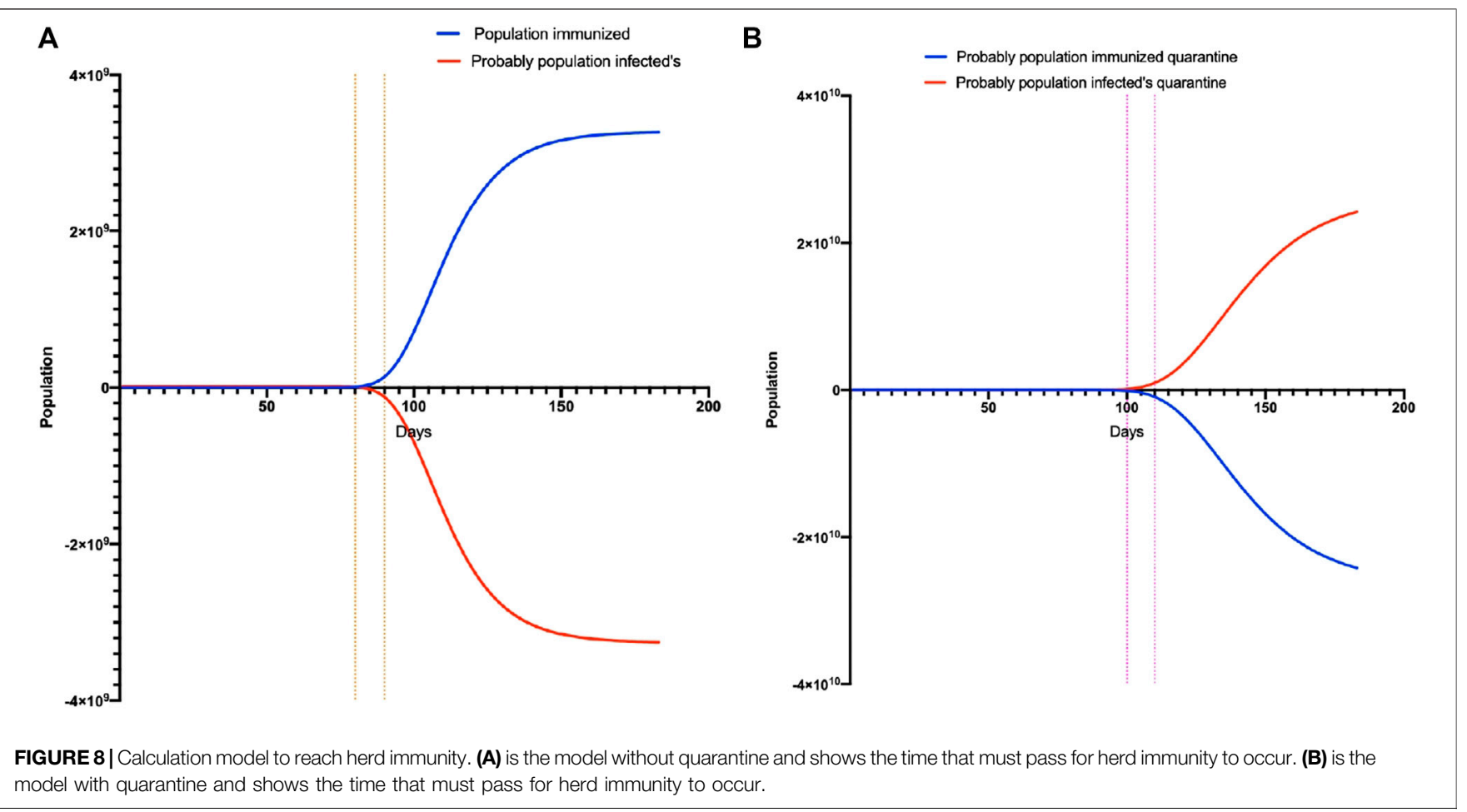

$R 0=2.2$, which is not too far from the international trend (Ye et al. estimated the same value of $R 0$, and the World Health Organization value is between 1.4 and 2.5).

The time of infection is generated based on $R 0$ (which depends on whether the value increases or decreases). If we compare the $R 0$ for other diseases caused by viruses of the same family (Middle East respiratory syndrome-related coronavirus, <1; SARS, 2-4), this value does not reflect the lethality for each one $[5,23,27,30$, 31 . Hence, defining $R 0$ is not easy and is mired in controversy. Each population could "fix" their value and reflect the variation in parameters of the equation used ( $\beta$ and $\alpha$ ) in each SIR model. The latter could be constructed and calculated in different ways depending on data reliability. However, the $R 0$ is very important because from it the minimum number of immunized people required to stop the epidemic, and the maximum number of individuals susceptible to infection, can be calculated [10, 11, 32, 33].

Some problems were identified when the $R 0$ was calculated on the basis of the total population. However, in reality, interventions are applied only in specific or priority groups. We analyzed different $R 0$ values for the COVID-19 epidemic in Ecuador and, despite an increase in $R 0,100 \%$ of a population will not be infected. With the prediction models used for smallpox prevention, the $R 0$ was 10 , but the maximum percentage of infected individuals in a vulnerable population was $70 \%$ and, upon introduction of a vaccine, any epidemic can be prevented with a coverage of $60 \%$ of the population $[6,16]$.

Complex and dynamic clinical phenomena occur during disease development. The immune response, nutritional state, chronic diseases, or co-infections can interfere (accelerate or decelerate) epidemic expansion, and are not taken into account when the $R 0$ is calculated [11]. Hence, $\mathrm{Li}$ and colleagues [11] postulated the interpretation and analyses of $R 0$ in four categories. Their model explains the rapid expansion of a disease but with a relatively slow recovery process.

$R t$ provides a clearer picture of the time of increase of an epidemic according to the percentage of susceptible people over time. Initially, the $R t$ shows an acceleration in the number of infected individuals, then becomes constant at the end of the epidemic, and reaches a value of 0 , thereby allowing calculation of an adjusted $R 0$ adapted to constant changes or population variation over time [33, 34]. A bottleneck has been observed in the $R t$ as the number of days of an epidemic increase, and the $R 0$ is closer to reality because, with fewer people to infect, the true infective capacity of a virus is observed. However, this scenario was not entirely accurate in our model $(R 0=1.97)$. Even though our $R 0$ is lower than that reported worldwide, some scholars have calculated an $R 0$ value between 0 and 1 . This does not mean that an epidemic is improving or ending; in some cases, this value shows a latency period [11, 32, 34, 35]. For a more accurate adjustment of the $R 0$, we applied Fso based on our obtained $R t$ value. As seen in Figure 7, the point where both curves intersect corresponds to the $R 0$ and simultaneously coincides with the bottleneck observed in $R t$ (Figure 5), and we showed a similar value of $R 0$ to that obtained in previous studies $[10,33]$.

The minimum number of infected people required to stop an epidemic has been mentioned $[9,23,33,36]$. However, this value must be considered very carefully because these infected individuals will probably die. Importantly, $60 \%$ of the population should be immunized (based on $R 0$ ) to stop the 
COVID-19 epidemic in Ecuador [9, 23, 33, 36]. This concept is seen more clearly with "collective immunity" or "collective protection", which refers to the fact that a group of infected people who recover will prevent the disease from spreading to the entire population $[21,22,37]$. By applying this concept to our SIR models, this collective protection will be reached with $60 \%$ of the infected population but, as stated by Rashid and colleagues, there are three main problems of assuming this value as real.

The first problem is the heterogeneity of the population (i.e., the different immune responses that each person presents to SARS-CoV-2). Hence, even though $60 \%$ of the population can be infected, this does not ensure that people can generate an immune state that protects the remaining susceptible members of the population $[35,37]$.

The second problem refers to the $R 0$ and the criterion of the equation for $\% P i, v$. Rashid et al. explained that, because the population is composed mostly of people at a high risk of contagion, the $R 0$ is overestimated. Hence, collective protection does not correspond to the one that has been calculated. In these cases, one must include in the equation the efficiency of the country's vaccination campaigns to understand how the epidemic can be controlled [20, 35, 37]. This was not applied in the present study because a vaccine for COVID-19 has not yet been found.

The third problem identified by Rashid et al. is the possible impact of co-circulation of other pathogens (depends on the characteristics of each population) during an epidemic. This could modified SIR models through the calculated $R 0$ with less or more impact in predictions, and not are fitted to reality.

We evaluated the impact of the quarantine implemented in Ecuador using two variables: the percentage of people quarantined (used in the SIR model) and $R t$ variation (by the effect of this measure). Quarantine reduces the number of people on the streets, and decreases the number of SARSCoV-2 infections in a particular period $[23,24]$. We took as a reference the percentage of people quarantined as $40 \%$. Other models have considered $10 \%$, and observed a decrease of $50 \%$ in the number of expected cases that would be observed if no health strategy was implemented. This $50 \%$ reduction does not mean that the cases will not exist, only that they will be redistributed over the duration of the epidemic. Hence, the epidemic would last 300 days due to the quarantine instead of 180-200 days, thereby ensuring that the health system does not collapse [29,30]. Although quarantine has been a good strategy for control of infectious diseases, it is not considered a good strategy from dynamic and economic viewpoints because external factors will affect Fso (the maximum number of susceptible people that can be infected). In our model, Fso increased by $10 \%$ (from 80 to $90 \%$ ) if quarantine was applied in $40 \%$ of the population. This increase occurred because the longer the quarantine lasts, the greater the probability that the population will not comply with it. Since quarantine was applied in Ecuador, the unemployment rate increased from 3.9 (2019) to $4.4 \%$ (2020). This increase forced people to take to the streets in search of work and increased the number of people exposed to SARS-CoV-2, and, consequently, Fso increased [24, 34, 38].
We also estimated the number of people with acquired immunity to SARS-CoV-2 based on the R0. We showed that a decrease in the number of newly infected cases would be observed by day 80 to day 100 of the epidemic. Other scholars say that this value depends on the responsiveness of the local government to report and identify cases, diagnostic capacity, and the delay in sample processing $[9,23,33,36]$.

We identified a delay in sample processing in $\geq 40 \%$ of cases, this parameter has not been reported before. Another value that can be estimated through the $R 0$ and Fso is the duration of infection. If $40 \%$ of the population were in quarantine, we estimated that the duration of infection would increase to 15 days, which was also observed by $\mathrm{Li}$ et al. and Massed et al. Both research teams agreed that the duration of infection and number of immunized individuals should not be taken into account to estimate the reduction of an epidemic. A latency period denotes the time when reinfections occur or the epidemic disappears. Hence, if we know the $R 0$, we could estimate the percentage of infected people required to stop the epidemic, which is equivalent to the one during this latent period. Our model determined it to be $65 \%$ but, in reality, this value will not be observed until it reaches $90 \%$ of infected people and only then will we observe a decrease in the infection rate [11, 23, 24, 33].

Although SIR models provide accurate data for the number of patients who have recovered from COVID-19, infected cases, and exposed patients, they require a large volume of reliable data. SIR models could be used in countries with better contingencies and strategies than those in most Latin American countries. Traditional models are dimensionless in time (results are not affected whether days or months are used) so the infection rate will not fit well with dynamic populations. For our models, we used a specific unit of time that allowed for the daily adjustment and discreet visualization of an increase or decrease of the epidemic. In the context of public health, the use of our model could enable prompt redistribution of resources according to infection trends.

A weakness of our models was not providing the percentage of asymptomatic cases and exposed cases. This led to a reduction of the power of prediction for infected people $(\geq 15 \%)$. Although this percentage could affect the fit between the real curve and model curve, this range of variation is acceptable because the exact proportion of asymptomatic COVID-19 individuals in Ecuador is not known. A lack of reliable and updated data was the main limitation of our models. This resulted from a deficiency in the structure of surveillance measurements taken, identification of cases, sample-processing capacity, as well as weakness in recollection, updating, and data analyses from the MSP during the COVID-19 epidemic. The MSP is the only entity that possesses full access to the data, and those data are not open for external analyses or validation. In addition, the number of reported cases and tests performed changed dramatically over time without robust justification from the MSP. Another important limitation was the assumption of some of the parameters for our models. They were obtained from countries from the same region with similar 
behavior of SARS-CoV-2 infection/COVID19 so did not affect our results.

\section{CONCLUSIONS}

For the entire Ecuadorian population, we estimated that $R 0=2.2$, with $88 \%$ susceptible/infected individuals. To stop a national epidemic, a quarantine for 3-4 months is required, and when $55 \%$ of the population has been immunized (equivalent to 110 days since the first report of a COVID-19 case), a real decrease of new cases will be observed. The effectiveness of quarantine should be analyzed retrospectively, and not as a result of contemporary control of the COVID-19 epidemic.

\section{REFERENCES}

1. Anthony SJ, Johnson CK, Greig DJ, Kramer S, Che X, Wells H, et al. Global patterns in coronavirus diversity. Virus Evolut (2017) 3(1):1-15. 10.1093/ve/ vex012.

2. Lal SK. Molecular biology of the SARS-coronavirus. In: Lal SK, editor Climate change 2013-the physical science basis. Vol. 53. Cambridge, United Kingdom: Cambridge University Press (2010) p. 1-30). doi:10.1017/СBO9781107415324. 004

3. Miller SL, Gill J, Webb GR. The proximal origin of the hamstrings and surrounding anatomy encountered during repair. J Bone Joint Surg (2007) 89(1):44-8. doi:10.2106/jbjs.f.00094.

4. Jin Y, Yang H, Ji W, Wu W, Chen S, Zhang W, et al. Virology, epidemiology, pathogenesis, and control of covid-19. Viruses (2020) 12(4):1-17. doi:10.3390/ v12040372.

5. Ye Q, Wang B, Mao J, Fu J, Shang S, Shu Q. Epidemiological analysis of COVID19 and practical experience from China. J Med Virol (2020) 12:23-7. doi:10. $1002 /$ jmv. 25813

6. Kaul D. An overview of coronaviruses including the SARS-2 coronavirus-molecular biology, epidemiology and clinical implications. Curr Med Res Pract (2020) 10(2):54-64. doi:10.1016/j.cmrp.2020.04.001.

7. Rothan HA, Byrareddy SN. The epidemiology and pathogenesis of coronavirus disease (COVID-19) outbreak. J Autoimmun (2020) 109, 102433. doi:10.1016/j. jaut.2020.102433.

8. SNGRE, CIES. Covid-19 en el ecuador tendencia temporo espacial de la pandemia del 27 de febrero al 19 de abril del 2020 INFORME. New York City, NY: MINTEL (2020)

9. MSP. Informes de Situación e Infografias-COVID 19-desde el 29 de Febrero del 2020-Servicio Nacional de Gestión de Riesgos y Emergencias. Quito, Ecuador: Ministerio de Salud Pública (2020)

10. Bjornstad ON. Epidemics models and data using R. Berlin, Germany: Springer (2010) doi:10.1007/978-3-319-97487-3

11. Li J, Blakeley D, Smith RJ. The failure of R0. Comput Math Methods Med (2011) 2011:1-17. doi:10.1155/2011/527610

12. Vynnycky E, White R. An introduction to infectious disease modelling. Oxford, UK: Oxford University Press (2010)

13. Keeling MJ, Rohani P. Modeling infectious diseases through Contact networks. Princeton, NJ: Princeton University Press (2008)

14. Li MY. An introduction to mathematical modeling of infectious diseases. In: L Ken M Golden editors. An introduction to mathematical modeling of infectious diseases. 2nd ed. Berlin, Germany: Springer International Publishing (2018) doi:10.1007/978-3-319-72122-4

15. NHCPRC. Latest developments in epidemic control. National Health Commission of the People's Republic of China (2020)

16. Jones JH. Notes on R 0. Building (2011) 1-19:17-33. doi:10.1186/ isrctn 10820810

17. Kretzschmar M. Measurement and modeling: infectious disease modeling. In: International encyclopedia of public health. 2nd ed. Vol.

\section{DATA AVAILABILITY STATEMENT}

The raw data supporting the conclusions of this article will be made available by the authors, without undue reservation.

\section{AUTHOR CONTRIBUTIONS}

PE and ET conceptualize the study, PE and PQ obtained data and analyzed it, PE set up the mathematical models, PE and PQ run the models, ET reviewed results and provided feedback. PE and PQ drafted the manuscript. ET drafted the final version. All authors revised and approved final version.

4. Amsterdam, Netherlands: Elsevier. doi:10.1016/B978-0-12-803678-5. 00229-0

18. Tarigan PB. Veterinary epidemiology. J Chem Inf Model (2013) 53(9):58-67. doi:10.1017/CBO9781107415324.004

19. Balkew TM. The SIR model when $S(t)$ is A multi-exponential function. East Tennessee State University (2010) $12 \mathrm{p}$.

20. Smith DR. Herd immunity. Vet Clin North Am Food Anim Pract (2019) 35(3): 593-604. doi:10.1016/j.cvfa.2019.07.001.

21. Chen J, Ye L, Zhou MY, Cheng YR, Wang M-W, Feng ZH. Herd immunity and COVID-19. Eur Rev Med Pharmacol Sci (2020) 24(8):4064-5. doi:10.26355/ eurrev_202004_20978.

22. Kwok KO, Lai F, Wei WI, Wong SYS, Tang JWT. Herd immunity-estimating the level required to halt the COVID-19 epidemics in affected countries. $J$ Infect (2020) 80(6):e32-3. doi:10.1016/j.jinf.2020.03.027.

23. Castro P, De los R, Juan C, González S, Merino P, Ponce J. Modelización y simulación de la propagación del virus sars-cov-2 en Ecuador. MODEMAT (2020) Available from: https://observatoriocovid19.sv/doc/biblioteca/internac/ Informe-Covid19-Modemat.pdf.

24. Aguirre H, Orellana D, Armas R. Escenarios de Propagación de COVID-19 al 21 de Abril y Relajamiento de Medidas de Mitigación. Cuenca, Ecuador: Universidad de Cuenca (2020) p. 1-14.

25. Cheng H-Y, Jian S-W, Liu D-P, Ng T-C, Huang W-T, Lin H-H. Contact tracing assessment of COVID-19 transmission dynamics in Taiwan and risk at different exposure periods before and after symptom onset. JAMA Intern Med (2020) 180:1156-63. doi:10.1001/jamainternmed.2020.2020.

26. INEC. Estadísticas Vitales registro estadístico de Nacidos vivos y Defunciones 2016. INEC (2016) 1, 315. doi:10.1016/j.jhazmat.2015.06.018

27. Bulut C, Kato Y. Epidemiology of COVID-19. Turk J Med Sci (2020) 50(SI-1), 563-70. doi:10.3906/sag-2004-172.

28. Petrosillo N, Viceconte G, Ergonul O, Ippolito G, Petersen E. COVID-19, SARS and MERS: are they closely related? Clin Microbiol Infect (2020) 22:74-8. doi:10.1016/j.cmi.2020.03.026

29. Stratton SJ. COVID-19: not a simple public health emergency. Prehosp Disaster Med (2020) 35(2):119. doi:10.1017/s1049023x2000031x.

30. Panovska-Griffiths J. Can mathematical modelling solve the current Covid19 crisis? BMC Publ Health (2020) 20(1):1-3. doi:10.1186/s12889-02008671-z.

31. Uras U. Coronavirus: comparing COVID-19, SARS and MERS|news|Al Jazeera (2020) Available from: https://www.aljazeera.com/news/2020/04/coronaviruscomparing-covid-19-sars-mers-200406165555715.html

32. Breban R, Vardavas R, Blower S. Theory versus data: how to calculate R0? PLoS One (2007) 2(3):e282. doi:10.1371/journal.pone.0000282.

33. Massad E. Ethical and Transborder issues. Global Health Inform (2017) 12 232-63. doi:10.1016/B978-0-12-804591-6.00012-4

34. Akpan N. How to measure your nation's response to coronavirus. London, UK: National Geographic (2020)

35. Metcalf CJE, Ferrari M, Graham AL, Grenfell BT. Understanding herd immunity. Trends Immunol (2015) 36(12):753-55. doi:10.1016/j.it.2015.10. 004 . 
36. Sun K, Chen J, Viboud C. Early epidemiological analysis of the coronavirus disease 2019 outbreak based on crowdsourced data: a population-level observational study. Lancet Dig Health (2020) 2:e201-8. doi:10.1016/S25897500(20)30026-1

37. Rashid H, Khandaker G, Booy R. Vaccination and herd immunity. Curr Opin Infect Dis (2012) 25(3):243-9. doi:10.1097/qco.0b013e328352f727

38. Lombeida E, Moreno L, Fabara C. Pobreza y desigualdad Ecuador (2010) Available from: http://www.ecuadorencifras.gob.ec/documentos/webinec/POBREZA/2018/Junio-2018/Informe_pobreza_y_desigualdad-junio_ 2018.pdf
Conflict of Interest: The authors declare that the research was conducted in the absence of any commercial or financial relationships that could be construed as a potential conflict of interest.

Copyright (c) Espinosa, Quirola and Teran. This is an open-access article distributed under the terms of the Creative Commons Attribution License (CC BY). The use, distribution or reproduction in other forums is permitted, provided the original author(s) and the copyright owner(s) are credited and that the original publication in this journal is cited, in accordance with accepted academic practice. No use, distribution or reproduction is permitted which does not comply with these terms. 